\title{
ZUR THEORIE DER LINEAREN SUBSTITUTIONEN
}

VON

E. NETTO

in GIESSEN.

Die Überführung einer linearen Substitution

$$
X_{\lambda}=\sum_{\mu} c_{\lambda \mu} x_{\mu}
$$

$(\lambda, \mu=1,2, \ldots, n)$

in ihre Normalform

$$
U_{\lambda}=\rho_{\lambda} u_{\lambda} \quad(\lambda=1,2, \ldots, n)
$$

ist ohne jede Schwierigkeit, sobald die charakteristische Gleichung

$$
\left|c_{\lambda \mu}-\rho \varepsilon_{\lambda \mu}\right|=0 \quad\left(\varepsilon_{\lambda \lambda}=\mathrm{I} ; \varepsilon_{\lambda \mu}=0 \text { falls } \lambda \neq \mu \text { ist }\right)
$$

nur verschiedene Wurzeln besitzt. Anders wird ès, wenn man diese Bedingung fallen lässt. Ich habe im Folgenden die Frage behandelt, ob es möglich ist, von der einfach herzustellenden Normalform einer nicht singulären Substitution zu der einer beliebigen benachbarten überzugehen, selbst in dem Falle, dass die charakteristische Gleichung der letzten mehrfache Wurzeln besitzt.

1.

In den Paragrafen I47-I57 seines Traité des substitutions giebt Herr C. Jondan eine »kanonische Form» der linearen Substitutionen von $n$ Veränderlichen. Dieselbe versagt aber, sobald die Determinante der Substitution verschwindet. Eine leichte Abänderung der Methode führt 
jedoch zu allgemeingültigen Ausdrücken. Das Resultat, welches sich dabei ergiebt, ist das folgende:

Es sei

$$
\Delta(\rho)= \pm\left(\rho-\rho_{1}\right)^{\sigma_{1}}\left(\rho-\rho_{2}\right)^{\sigma_{2}} \ldots\left(\rho-\rho_{\tau}\right)^{\sigma_{\tau}} \quad\left(\rho_{\alpha} \neq \rho_{\beta} ; 2 \sigma=n\right)
$$

die charakteristische Function der Substitution

$$
X_{\lambda}=\sum_{\mu} c_{\lambda \mu} x_{\mu} \quad(\lambda, \mu=1,2, \ldots, n)
$$

dann lässt diese sich durch die Einführung linearer homogener Verbindurgen der $x_{\mu}$ als neuer Veränderlichen auf die Form bringen

$$
\begin{array}{lll}
U_{1}^{\prime}=\rho_{1} u_{1}^{\prime}, & U_{1}^{\prime \prime}=u_{1}^{\prime}+\rho_{1} u_{1}^{\prime \prime}, \ldots, \quad U_{1}^{(\pi)}=u_{1}^{(\pi-1)}+\rho_{1} u_{1}^{(\pi)}, \\
U_{2}^{\prime}=\rho_{1} u_{2}^{\prime}, & U_{2}^{\prime \prime}=u_{2}^{\prime}+\rho_{1} u_{2}^{\prime \prime}, \ldots, \quad U_{2}^{\left(\pi_{2}\right)}=u_{2}^{\left(\pi_{2}-1\right)}+\rho_{1} u_{2}^{\left(\pi_{2}\right)},
\end{array}
$$

$$
\begin{aligned}
& U_{h_{1}^{\prime}}^{\prime}=\rho_{1} u_{h_{1}}^{\prime}, \quad U_{h_{1}}^{\prime \prime}=u_{h_{1}}^{\prime}+\rho_{1} u_{h_{1}}^{\prime}, \ldots \\
& V_{1}^{\prime}=\rho_{2} v_{1}^{\prime}, \quad V_{1}^{\prime \prime}=v_{1}^{\prime}+\rho_{2} v_{1}^{\prime \prime}, \quad \ldots ., \quad V_{1}^{(\tau)}=v_{1}^{(\tau-1)}+\rho_{2} v_{1}^{(\tau)} \text {, } \\
& \left(\pi+\pi_{2}+\ldots+\pi_{h_{1}}=\sigma_{1}, \tau+\tau_{2}+\ldots+\tau_{h_{2}}=\sigma_{2}, \ldots\right) .
\end{aligned}
$$

Schreibt man jetzt die charakteristische Determinante der Substitution, die ja durch die Einführung der $u, v, \ldots$ keine Änderung erfahren hat, in ihrer zu (3) gehörigen Form nieder, so enthält sie nur in der Hauptdiagonale und in der dazu parallelen, links benachbarten Reihe von Null verschiedene Elemente. In der Hauptdiagonale steht zuerst $\sigma_{1}$-mal das Glied $\rho_{1}-\rho$, dann $\sigma_{2}$-mal $\rho_{2}-\rho$, u. s. f. In der links benachbarten Parallelreihe steht in allen zu $U_{a}^{\prime \prime}, U_{a}^{\prime \prime \prime}, \ldots, V_{a}^{\prime \prime}, V_{a,}^{\prime \prime \prime}, \ldots$ gehörigen Zeilen eine I, sonst überall eine Null.

Die Glieder $u_{\alpha}$ oder $v_{\alpha} \ldots$, die in (3) einer und derselben Zeile angehören, wollen wir einer Kette der Normalform zurechnen; die Anzahl der Glieder einer Kette werde als ihre Ordnung bezeichnet; die Ausdrücke $u, v, \ldots$ wollen wir die Normalcoordinaten nennen.

Erwahnt sei der besondere Fall, in welchem die charakteristische Function die Form $\rho^{n}$ annimmt. Hier erhält man

$$
U_{1}=0, \quad U_{2}=\varepsilon_{2} u_{1}, \quad U_{3}=\varepsilon_{3} u_{2}, \ldots, \quad U_{n}=\varepsilon_{n} u_{n-1},
$$


wobei die $\varepsilon$ beliebig einen der Werte o, I annehmen können. Daraus lässt sich dann, wie leicht zu sehen ist, die allgemeine Form von (2) angeben. Es sei

$$
u_{\lambda}=\sum_{\mu} a_{\lambda, \mu} x_{\mu}, \quad x_{\lambda}=\sum_{\mu} \alpha_{\lambda, \mu} u_{\mu}, \quad(\lambda, \mu=1,3, \ldots, n)
$$

dann hat man, falls $\varepsilon_{1}=0$ und $u_{0}=0$ gesetzt wird

$$
X_{\lambda}=\sum_{\mu} \alpha_{\lambda, \mu} U_{\mu}=\sum_{\mu} \alpha_{\lambda \mu} \varepsilon_{\mu} u_{\mu-1}=\sum_{\mu, \nu} \alpha_{\lambda, \mu} a_{\mu-1, \nu} \varepsilon_{\mu} x_{\nu} \quad\left(\begin{array}{c}
\lambda, \nu=1,2, \ldots, n \\
\mu=1,2, \ldots, n \\
\varepsilon_{1}, \varepsilon_{2}=0,1
\end{array}\right)
$$

als allgemeine Form derjenigen Substitutionen, die bei mehrfacher Wiederholung schliesslich alle Variablen zu Null machen.

\section{2.}

Wir behandeln jetzt die Aufgabe, die Elementarteiler bei der zu $\S$ I, (3) gehörigen Determinante der Normalform zu bestimmen. Sie sind nach der Einführung des Herrn Weienstrass folqendermassen zu definiren: Es sei $\left(o_{\alpha}-\rho\right)^{p_{r}}$ die höchste Potenz von $\left(o_{a}-\rho\right)$, welche alle ${ }^{\text {ten }}$ Subdeterminanten, d. h. alle von der Ordnung $n-r$ teilt, dann werden

$$
\left(\rho_{a}-\rho\right)^{p_{0}-p_{1}},\left(\rho_{a}-\rho\right)^{p_{1}-p_{2}},\left(\rho_{a}-\rho\right)^{p_{2}-p_{3}}, \ldots
$$

die zu $\left(\rho_{a}-\rho\right)$ gehörigen Elementarteiler.

Um diese in unserem Falle zu bestimmen, bedarf es einiger Voruntersuchungen.

Wir betrachten zuerst die Subdeterminanten der zu $\S$ I, (3) gehörigen Determinante $\Delta$, und bezeichnen sie durch

$$
\Delta_{a_{1} \beta_{1}, a_{w} \beta_{22}, \ldots, a_{r} \beta_{r}},
$$

wobei die ersten Indices sich auf die Ordnung der weggelassenen Zeilen $\left(Z_{o_{1}}, Z_{o_{2}}, \ldots, Z_{a_{\mathrm{r}}}\right)$, die zweiten Indices $\beta$ sich auf die Ordnung der weggelassenen Colonnen $\left(C_{\beta_{1}}, C_{\beta_{2}}, \ldots, C_{\beta_{r}}\right)$ beziehen. Dabei können wir natürlich voraussetzen

$$
\alpha_{1}<a_{2}<\alpha_{3}<\ldots ; \quad \beta_{1}<\beta_{2}<\beta_{3}<\ldots
$$


E. Netto.

Dann erkennt man zunächst, dass (2) identisch verschwindet, falls $\beta_{1}<\alpha_{1}$ ist. Denn tilgt man in unserer Determinante die Colonne $C_{\beta_{1}}$, dann treten in die zurückbleibende Matrize von $n(n-1)$ Gliedern, welche für den Augenblick mit $d_{a^{3}}$ bezeichnet werden sollen, für die Elemente

$$
\begin{array}{llll}
d_{1, \beta_{1}} & d_{1, \beta_{1}+1} & \ldots & d_{1, n-1} \\
d_{2, \beta_{1}} & d_{2, \beta_{1}+1} & \ldots & d_{2, n-1} \\
\cdots & \ldots & \ldots & \ldots \\
d_{\beta_{1}, \beta_{1}} & d_{\beta_{1}, \beta_{3}+1} & \ldots & d_{\beta_{1}, n-1}
\end{array}
$$

nur Nullen ein; denn alle diese Elemente standen in $\Delta$ rechts von der Diagonale. Tilgt man dann die weiteren Zeilen und Colonnen, so wird, wenn $\alpha_{1}>\beta_{1}$ ist, immer noch ein System von $\beta_{1}\left[(n-r)-\left(\beta_{1}-\mathrm{I}\right)\right]$ Nullen zurückbleiben, die in $\beta_{1}$ Zeilen zu je $(n-r)-\left(\beta_{1}-1\right)$ angeordnet sind. Das genügt aber, um (2) zum Verschwinden zu bringen.

Soll also (2) von Null verschieden sein, so ist $\alpha_{1} \leqq \beta_{1}$ zu setzen.

Ferner verschwindet (2), wenn $\beta_{1} \geq \alpha_{2}$ ist. Denn wenn $\alpha_{1}<\alpha_{2} \leqq \beta_{1}$ angenommen wird, dann entsteht aus $\Delta$ nach der Tilgung von $Z_{\alpha_{1}}, Z_{v_{2}}$ eine Matrize von $n(n-2)$ Gliedern, welche wiederum für den Augenblick mit $d_{a, \beta}$ bezeichnet werden mögen. In ihr werden die Elemente

$$
\begin{array}{llll}
d_{\alpha_{2}, 1} & d_{\alpha_{2}, 2} & \ldots & d_{a_{2}, \alpha_{2}} \\
d_{a_{2}+1,1} & d_{a_{2}+1,2} & \ldots & d_{a_{2}+1, a_{2}} \\
\ldots & \ldots \ldots & \ldots \\
d_{n-2,1} & d_{n-2,2} & \ldots & d_{n-2, \alpha_{2}}
\end{array}
$$

sämmtlich gleich Null, da sich alle diese in $\Delta$ links von der ersten $\mathrm{Pa}$ rallelreihe zur Hauptdiagonale befanden. Tilgt man nun die übrigen Zeilen und Colonnen, so bleiben, falls $\beta_{1}>\alpha_{2}$ ist, immer noch

$$
\left[(n-r)-\left(\alpha_{2}-1\right)\right] \alpha_{2}
$$

Nullen in $\alpha_{2}$ Colonnen zu je $(n-r)-\left(\alpha_{2}-r\right)$ verteilt zurück, und dadurch wird das Verschwinden von (2) bewirkt. Ist aber $\beta_{1}=\alpha_{2}$, so betrachten wir statt (4) das System von Nullen 


$$
\begin{array}{llll}
d_{a_{2}-1,1} & d_{a_{2}-1,2} & \ldots & d_{a_{2}-1, \alpha_{2}-1} \\
d_{a_{2,1}, 1} & d_{n_{2,2}} & \ldots & d_{a_{2,}, a_{2}-1} \\
\ldots & \ldots \ldots & \ldots \\
d_{n-2,1} & d_{n-2,2} & \ldots & d_{n-2, \alpha_{2}-1}
\end{array}
$$

und dies führt nach Tilgung der übrigen vorgeschriebenen Zeilen und Colonnen auf ein System von $\left[(n-1)-\left(x_{2}-2\right)\right]\left(x_{2}-1\right)$ Nullen in (2). Diese Determinante verschwindet also auch hier.

Will man demnach das identische Verschwinden von (2) vermeiden, so muss man $\alpha_{1} \leq \beta_{1}<\alpha_{2}$ annehmen.

Unterdrückt man darauf, um (2) zu bilden in $\Delta(\rho)$ die beiden Reihen $Z_{a_{1}}, C_{\beta_{1}}$, so entsteht zunächst eine Matrix von $(n-1)(n-1)$ Elementen, die für den Augenblick wieder mit $d_{a, \gamma^{3}}$ bezeichnet werden mögen. In ihr ist jedes Element $\lambda_{\lambda, \mu}$ bei dem $\lambda \leq \mu, \mu>\beta_{1}$ oder bei dem $\alpha>\beta_{1}, \mu \leq \beta_{1}$ ist, gleich Null. Folglich zerfällt $\Delta_{a_{1, \beta_{1}}}$ in das Product aus einer Determinante $\left(\beta_{1}-1\right)^{\text {ter }}$ und einer solchen $\left(n-\beta_{1}\right)^{\text {ter }}$ Ordnung. Jene erste bleibt bei den weiteren Reihenstreichungen, die auf ( 2 ) führen, unberührt. Die zweite hat dieselbe Bildung wie $\Delta(o)$ und es gelten daher für den Übergang zunächst zu $\Delta_{a_{1} \xi_{1}, \alpha_{\alpha} \beta_{2}}$ dieselben Überlegungen. Somit erkennt man:

Damit (2) von Null verschieden sei, müssen die Beziehungen gelten

$$
\alpha_{1} \leqq \beta_{1}<\alpha_{2} \leqq \beta_{2}<\alpha_{3} \leqq \beta_{3}<\ldots
$$

Findet dies statt, dann zerfält, wie sich soeben gezeigt hat, (2) in ein Product von Subdeterminanten, deren jede ihrem Hauptgliede gleich wird, da bei dem einen Teile derselben rechts, bei dem anderen links von der Diagonale nur Nullen stehen. Das Erste findet bei dem ersten, dritten, fünften, ... Factor statt; diesen kommen die Gradzahlen $\left(\alpha_{1}-1\right)$, $\left(\alpha_{2}-1-\beta_{1}\right),\left(\alpha_{3}-1-\beta_{2}\right), \ldots z u$. Das zweite findet bei dem zweiten, vierten, sechsten, ... Factor statt; diesen kommen die Gradzahlen der Determinanten $\left(\beta_{1}-\alpha_{1}\right),\left(\beta_{2}-\alpha_{2}\right),\left(\beta_{3}-\alpha_{3}\right), \ldots$ zu. Die Determinanten der ersten Art haben in der Hauptdiagonale nur Elemente $\left(\rho_{a}-\rho\right)$ und ihre Werte werden sonach Potenzen von solchen Differenzen. Die Determinanten der zweiten Art haben in der Hauptdiagonale nur Grössen 
I oder o. Eine Null tritt dann und nur dann auf, wenn eine der erwähnten Partialdeterminanten aus einer der Ketten in die folgende hinüber greift; denn die der Hauptdiagonale benachbarte linke Parallelreihe, welche hier in die Diagonalreihe der zweiten, vierten,... Partialdeterminante gerückt ist, enthält stets und auch nur bei Beginn einer Kette das Glied o. Es ergiebt sich daher weiter:

Damit (2) nicht verschwinde, ist ferner nötig, dass $Z_{\alpha_{1}}$ und $C_{\beta_{1}}$, ebenso $Z_{a_{2}}$ und $C_{\beta_{2}}, \ldots$ jedesmal solche Diagonalglieder aus $\Delta(\rho)$ ausschneiden, welche derselben Ketie angehören.

Nach diesen Untersuchungen ist es leicht, die zu $\rho_{\alpha}-\rho$ gehörigen Elementarteiler zu bestimmen. Dabei muss die Gesammtheit der nicht verschwindenden $\Delta_{a_{1} \beta_{1}}$ betrachtet werden. Wählt man $Z_{a_{1}}, C_{\beta_{1}}$ so, dass die Diagonalglieder, welche $\Delta_{\alpha_{1} \hat{\beta}_{1}}$ weniger hat als $\Delta$, einer zu $\rho_{1}$ gehörigen Kette entnommen sind, dann tritt für die zweite Partialdeterminante der zweite, soeben erwähnte Fall ein. Es werden $\beta_{1}-\alpha_{1}$ von den $\sigma_{1}$ Elementen $\rho_{1}-\rho$, die in der Diagonale von $\Delta(\rho)$ vorkommen, jetzt ausscheiden. Nimmt man also $\alpha_{1}=\mathrm{I}, \beta_{1}=\pi$, dann fehlt die i. A. erste Determinante ganz; die zweite, hier an erster Stelle auftretende gehört dem zweiten Typus an, und so fält, als höchst mögliche Potenz $\left(\rho_{1}-\rho\right)^{\pi-1}$ fort, und $\Delta_{a_{1} \beta_{1}}$ enthält $\left(\rho_{1}-\rho\right)$ nur noch in der Potenz $\left(\sigma_{1}-1\right)-(\pi-1)=\sigma_{1}-\pi$. Eine höhere Potenz kann nicht in Wegfall kommen, ohne dass $\Delta_{\omega_{1} \beta_{1}}=0$ wird. Die Vergleichung mit (I) zeigt, dass $p_{0}=\sigma_{1}, p_{1}=\sigma_{1}-\pi$ wird, und dass also $\pi$ der Exponent des ersten Elementartcilers ist. Diese Potenz von $\rho_{1}-\rho$ ist die höchste, welche alle Subdeterminanten $\Delta_{a_{1} \beta_{3}}$ von $\Delta$ teilt.

Un aus $\Delta_{a_{1}, \hat{F}_{1}, \alpha_{2} \beta_{2}}$ eine möglichst hohe Potenz von $\rho_{1}-\rho$ ausscheiden zu können, müssen die beiden entsprechenden Partialdeterminanten zweiter Art möglichst hohe Potenzen von $\rho_{1}-\rho$ enthalten; dies findet statt, wenn man $\alpha_{1}=1, \beta_{1}=\pi ; \alpha_{2}=\pi+1, \beta_{2}=\pi+\pi_{2}$ setzt. Dann erhält man, da zwei Determinanten erster Art von den Ordnungen

$$
\alpha_{1}-\mathrm{I}=\mathrm{o}, \quad \alpha_{2}-\mathrm{I}-\beta_{1}=0
$$

vorhanden sind, $\left(\rho_{1}-\rho\right)$ in der Potenz $\sigma_{1}-\pi-\pi_{2}$. Dies ist gemäss (I) gleich $p_{2}$ zu setzen, und dann wird $p_{1}-p_{3}=\pi_{2}$. Geht man in dieser Weise fort, so erhalt man das Resultat: 
Zur Theorie der linearen Substitutionen.

Die Determinante $\Delta(\rho)$ erscheint, in ihre Elementarteiler aufgelöst, unter der Form

$$
\Delta(\rho)= \pm\left(\rho_{1}-\rho\right)^{\pi}\left(\rho_{1}-\rho\right)^{\pi_{2}}\left(\rho_{1}-\rho\right)^{\pi_{3}} \cdots
$$

$$
\left(\rho_{2}-\rho\right)^{\tau}\left(\rho_{2}-\rho\right)^{\tau_{2}}\left(\rho_{2}-\rho\right)^{\tau_{3}} \ldots
$$

so dass die Ordnungen der Ketten, ihrer Grösse nach geordnet, als Exponenten der aufeinanderfolgenden Elementarteiler auftreten.

3.

Wir wollen jetzt von der Substitution (2) § I mit den Coefficienten $c_{\lambda, \mu}$ zu einer neuen Substitution dadurch übergehen, dass wir jedem Elemente $c_{\lambda \mu}$ noch additiv $\gamma_{\lambda_{\mu}}, t$ hinzufügen. Die $\gamma$ sollen dabei beliebige endliche Constanten sein, während $t$ eine unendlich kleine Veränderliche ist. Es folgt leicht, dass die Wahl der $\gamma$ so getroffen werden kann, dass

$$
\theta(\rho)=\theta=\left|c_{x \lambda}+\gamma_{x \lambda} t-\rho \varepsilon_{x \lambda}\right|=0
$$

für alle von Null verschiedenen, hinlänglich kleinen Werte $t$ nur ungleiche Wurzeln besitzt. Führt man die erste Substitution durch die Benutzung der Normalcoordinaten $u_{x}=\sum_{a_{x \lambda}} x_{\lambda}$ in die Normalform über, so wird die gleichzeitige Einführung der $u$ in die zweite Substitution diese in eine Form bringen, welcher jene Normalform benachbart ist, und Coefficienten besitzen, welche in $t$ linear sind.

Wir wollen die Wurzeln von $\theta=0$ untersuchen, aber nicht gleich in voller Allgemeinheit, sondern zunächst in dem Falle, dass $\Delta(\rho)=\left(\rho_{1}-\rho\right)^{n}$ wird. Es ist ferner nur eine Erleichterung in der Bezeichnung, wenn wir $\rho_{1}=\mathrm{O}$ setzen. Wir wollen annehmen, dass in der zu $\theta$ gehörigen Substitution $k$ Ketten, bezw. von der Ordnungen

$$
h_{1} \geq h_{2} \geq h_{3} \geq \cdots \geq h_{k}
$$

vorhanden sind; alle Diagonalglieder in $\theta$ haben die Form $\gamma_{x x} t-\rho$; ferner ist, wenn die Glieder in $\theta$ mit $d_{x \lambda}$ bezeichnet werden; 


$$
\begin{gathered}
d_{21}=r_{21} \cdot t+\mathrm{I}, \quad d_{32}=r_{32} \cdot t+\mathrm{I}, \ldots, \quad d_{h_{1}, h_{1}-1}=r_{h_{1}, h_{1}-1} \cdot t+\mathrm{I}, \\
d_{h_{1}+1, h_{1}}=r_{h_{1}+1, h_{1}} \cdot t, \quad d_{h_{1}+2, h_{1}+1}=r_{h_{1}+2, h_{1}+1} \cdot t+\mathrm{I}, \ldots, \\
d_{h_{1}+h_{2}, h_{1}+h_{2}-1}=r_{h_{1}+h_{2}, h_{1}+h_{2}-1} \cdot t+\mathrm{I}, \\
d_{h_{1}+h_{2}+1, h_{1}+h_{2}}=r_{h_{1}+h_{2}+1, h_{1}+h_{2}} \cdot t, \quad d_{h_{1}+h_{2}+2, h_{1}+h_{2}+1}=r_{h_{1}+h_{2}+2, h_{1}+h_{2}+1} \cdot t+\mathrm{I}, \ldots,
\end{gathered}
$$

wăhrend die übrigen $d_{x \lambda}$ alle gleich den $\gamma_{x \lambda}, t$ zu setzen sind.

Entwickelt man nun $\theta(\rho)$ nach Potenzen von $\rho$, dann wird $\pm \rho^{n-a}$ mit der Summe aller der Subdeterminanten $\alpha^{\text {ter }}$ Ordnung von $\theta(0)$ multiplicirt werden, deren Hauptdiagonalen der Hauptdiagonale von $\theta(0)$ entnommen sind. Es kommt uns darauf an, die niedrigste, in dieser Summe vorkommende Potenz von $t$ zu ermitteln.

Für $a=\mathrm{I}$ finden wir sofort $t^{1} \sum_{\gamma_{\lambda \lambda} \text {. }}$.

Für $a=2$ betrachten wir, wenn $h_{1}>_{1}$ ist, die Determinante

$$
\left|\begin{array}{ll}
r_{11} t & r_{12} t \\
r_{21} t+\mathbf{I} & r_{22} t
\end{array}\right|
$$

und sehen, dass auch hier $t^{1}$ das niedrigste auftretende Glied ist.

Für $\alpha=3$ folgt, wenn $h_{1}>2$ ist, das gleiche Resultat, wie aus

hervorgeht.

$$
\left|\begin{array}{lll}
r_{11} t & r_{12} t & r_{13} t \\
r_{21} t+\mathrm{I} & r_{22} t & r_{23} t \\
r_{31} t & r_{32} t+\mathrm{I} & r_{33} t
\end{array}\right|
$$

Das setzt sich so fort, bis zu $a=h_{1}$, wo in der entsprechend gebildeten Determinante noch $t^{1}$ auftritt.

Nimmt man aber weiter $\alpha=h_{1}+\mathrm{I}$, so sieht man unmittelbar, dass $t^{1}$ nicht mehr auftreten kann. Denn dies ist nur möglich, wenn in einer der als Coefficienten auftretenden Determinanten alle Glieder der zur Hauptreihe links benachbarten Reihe den Summanden I enthalten; das. geht hier aber nicht mehr, da keine Kette von hinreichend hoher Ordnung ist. Andererseits erkennt man aus der Determinante $\left|d_{x \lambda}\right|$, $\left(x, \lambda=\mathrm{I}, 2, \ldots, h_{1}+\mathrm{I}\right)$, dass ein Glied mit $t^{2}$ auch wirklich erscheint. 
Zur Theorie der linearen Substitutionen.

In gleicher Art folgt für $x, \lambda=\mathbf{I}, 2, \ldots, h_{1}+2$, dann für $x, \lambda=\mathrm{I}, 2, \ldots, h_{1}+3$, u. s. f. bis zu $x, \lambda=1,2, \ldots, h_{1}+h_{2}$, dass jedesmal Glieder mit $t^{2}$ vorhanden sind.

Für $a=h_{1}+h_{2}+\mathrm{r}$ bis $\alpha=h_{1}+h_{2}+h_{3}$ giebt es Glieder mit $t^{3}$, u. s. f.

Es wird daher die Entwicklung die folgende werden:

$$
\begin{aligned}
& \theta(\rho)= \pm \rho^{n}+A_{1} t \rho^{n-1}+A_{2} t \rho^{n-2}+\ldots+A_{h_{1}} t \rho^{n-n_{1}} \\
& +B_{1} t^{2} \rho^{n-h_{1}-1}+B_{2} t^{2} \rho^{n-h_{1}-2}+\ldots+B_{h_{2}} t^{2} \rho^{n-h_{1}-h_{2}} \\
& +F_{1} t^{k} \rho^{h_{k}-1}+F_{2} t^{k} \rho^{h_{k}-2}+\ldots+F_{h_{k}} t^{k}
\end{aligned}
$$

die Exponenten der letzten Zeile folgen aus

$$
h_{1}+h_{2}+h_{3}+\ldots+h_{k}=n .
$$

Freilich ist bisher nur gezeigt worden, dass die angegebenen Potenzen von $t$ die niedrigsten sind, die überhaupt vorkommen können, aber noch nicht, dass sie auch wirklich auftreten. Dies zeigen wir am einfachsten an folgendem Beispiele: Es ist, wie man ohne Schwierigkeit erkennt,

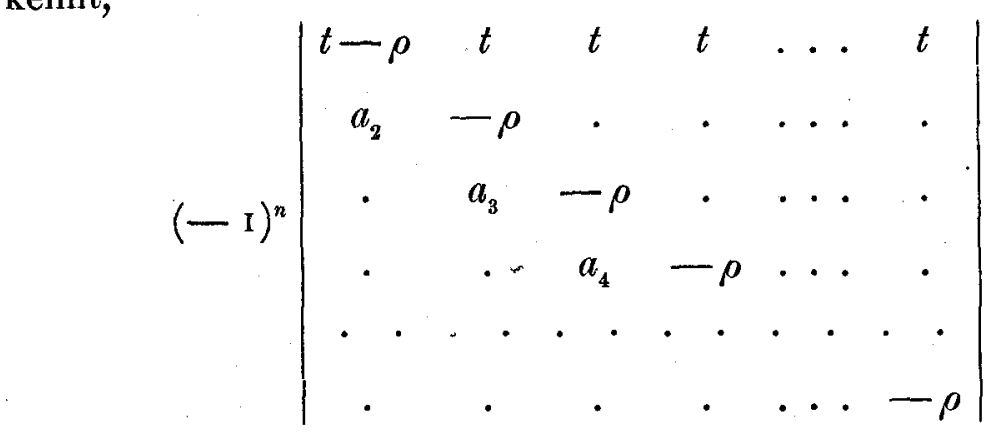

$$
\begin{aligned}
& =\rho^{n}-t \rho^{n-1}-a_{2} t \rho^{n-2}-a_{2} a_{3} t \rho^{n-3}-a_{2} a_{3} a_{4} t \rho^{n-4}-\ldots-a_{2} a_{3} \ldots a_{n} t
\end{aligned}
$$

Setzt man hierin die $a_{\lambda}$ gleich den $d_{\lambda, \lambda-1}$ aus (I), dann liefert diese Determinante ein Beispiel für $\theta(\rho)$, bei welchem die Form (2) wirklich auftritt. Dieselbe Form muss daher für unbestimmte $r$ bestehen.

Nachdem diese erste Frage entschieden ist, wollen wir an zweiter Stelle die Wurzelentwickelungen für $\theta(\rho)=0$ gemäss (2) vornehmen. Dies gestaltet sich bei der Anwendung des sogenannten NewTon'schen 
Parallelogramms ${ }^{1}$ besonders einfach. Es handelt sich hierbei darum, die Glieder zu bestimmen, welche auf der rechten Seite von (2) die niedrigste Dimension erhalten, wenn man für $\rho$ etwa $t^{a}$ einsetzt, und darum, die passenden Werte von $\alpha$ anzugeben. Dabei kommen ausser $\rho^{n}$ sicher nur die Schlussglieder der einzelnen Zeilen in Frage, also

$$
t^{k}, t^{k-1+\alpha h_{k}}, t^{k-2+\alpha\left(h_{k}+h_{k-1}\right)}, t^{\left.k-3+\alpha_{i}^{\prime} h_{k}+h_{k-1}+h_{k-2}\right)}, \ldots, t^{a n} .
$$

Hier ist zu beachten, dass man hat

$$
h_{k} \leqq h_{k-1} \leqq h_{k-2} \leqq \cdots \leqq h_{1}
$$

daraus folgt, dass für

$$
\alpha=\frac{\mathrm{I}}{h_{k}} \quad \text { die beiden ersten Exponenten; für }
$$

$$
\begin{aligned}
& \alpha=\frac{1}{h_{k-1}} \text { der zweite und der dritte Exponent; für } \\
& \alpha=\frac{1}{h_{k \rightarrow 2}} \quad \text { der dritte und der vierte Exponent u. s. w. }
\end{aligned}
$$

die niedrigsten Werte annehmen. Dieses Verhältnis wird auch dann nicht gestört, wenn mehrere der $h$ einander gleich werden; dann werden eben nur die entsprechenden $a$ und die Exponenten selbst einander gleich.

Nach dem NEwTov'schen Satze ergeben sich dann die Entwickelungen

$$
\begin{array}{ll}
\rho_{1}=\mathfrak{P}_{1}\left(t^{\frac{1}{h_{1}}}\right), & \rho_{2}=\mathfrak{P}_{1}\left(\omega_{1}^{1} t^{\frac{1}{h_{1}}}\right), \ldots, \quad \rho_{h_{1}}=\mathfrak{P}_{1}\left(\omega_{1}^{h_{1}-1} t^{\frac{1}{h_{1}}}\right), \\
\rho_{h_{1}+1}=\mathfrak{P}_{1}\left(t^{\frac{1}{h_{2}}}\right), & \rho_{h_{1}+2}=\mathfrak{P}_{2}\left(\omega_{2}^{1} t^{\frac{1}{h_{2}}}\right), \ldots, \rho_{h_{1}+h_{2}}=\mathfrak{P}_{2}\left(\omega_{2}^{h_{2}-1} t^{\frac{1}{h_{2}}}\right),
\end{array}
$$

wobei $\mathfrak{P}_{1}, \mathfrak{P}_{2}, \ldots$ Potenzreihen bedeuten, die nach ganzen Potenzen der

1 Vgl. Balitzi:R: Analytische Geometrie, S. 305. PuIseUx: Untersuchungen über die algebraischen Functionen, dargestellt von H. Fischer, S. I 5 ff. 
Argumente fortschreiten, und die $\omega_{1}, \omega_{2}, \ldots$ primitive $h_{1}^{\text {te }}, h_{2}^{\text {te }}, \ldots$ Einheitswurzeln sind.

Gleiches folgt für den allgerneinen Fall, sobald die Potenzen (3) auch bei der allgemeinen Determinante als diejenigen der niedrigsten Ordnungen bestehen bleiben. Es ist auf die folgende Art leicht zu sehen, dass dies tatsächlich eintrifft.

Wir wollen eine Substitution $(n+m)^{\text {ter }}$ Ordnung in der Normalform annehmen; $\rho=0$ sei wie bisher die $n$-fache Wurzel; die übrigen Wurzeln mögen $\rho_{1}, \rho_{2}, \ldots$ heissen. Wir bilden wie vorher die Determinante $\Delta_{1}(\rho)$ der benachbarten Substitution. Ihre ersten $n$ Zeilen stimmen in ihren ersten $n$ Colonnen mit dem $\theta(\rho)$, welches wir soeben untersucht haben, überein. Wir entwickeln nun $\Delta_{1}(\rho)$ dem Laplace'schen Satze gemäss in eine Summe von Producten aus Determinanten, die den $n$ ersten Zeilen, und solchen, die den $m$ letzten entnommen sind. Dabei erhält man zunächst $\theta(\rho)$ mit einer Determinante $\Delta_{2}(\rho)$ der $m^{\text {ten }}$ Ordnung multiplicirt. Diese beginnt mit einer Constanten bei ihrer Entwickelung nach Potenzen von $\rho$; so dass also sämmtliche in (2) enthaltenen Glieder wieder auftreten; und diese sind in $\theta(\rho) \cdot \Delta_{2}(\rho)$ die der niedrigsten Dimension, da jede Multiplication von (2) mit einem $\rho^{\tau} t^{\sigma}$ nur die Dimension erhöht. Ausser dem 'betrachteten Producte $\theta(\rho) \cdot \Delta_{2}(\rho)$ kommt eine Reihe ähnlicher Glieder vor, die aus jenem durch Austausch von Colonnen entstehen. Hierbei fallen aber in jedem Factor höchstens Glicder, die gleich I sind, aus der Diagonal-Parallelreihe fort, d. h. es treten allenfalls höhere aber niemals niedere Potenzen von $t$ bei denselben Potenzen von $\rho$ auf. Die Dimension der Glieder vermindert sich daher nicht.

Ferner liefern die Annahmen (4) genau die $n$ Wurzeln, welche für $t=\mathrm{o} \mathrm{zu} \rho=\mathrm{o}$ werden; weitere Wurzeln und weitere, hierher gehörige Glieder niedrigster Dimension giebt es also nicht.

Folglich gelten die über die Wurzelentwickelung g̀omachten Schlüsse.

Ist eine Substitution mit den Coefficienten $c_{x \lambda}$ gegeben, welche $\rho=\rho_{0}$ als Wurzel der charakteristischen Gleichung besitzt, und bildet man eine Substitution mit den Coefficienten $c_{x \lambda}+r_{x \lambda} . t$, worin $\gamma_{x \lambda}$ unbestimmte Constanten und $t$ eine beliebig kleine Veränderliche bedeuten, so möge es für

$$
\Delta\left(c_{x \lambda}+\gamma_{x \lambda} t-\rho \varepsilon_{x \lambda}\right)=0
$$


die Entwickelungen

$$
\begin{array}{r}
\rho_{1}-\rho_{0}=\mathfrak{P}_{1}\left(t^{\frac{1}{h_{2}}}\right), \quad \rho_{2}-\rho_{0}=\mathfrak{P}_{1}\left(\omega_{1}^{1} t^{\frac{1}{h_{1}}}\right), \ldots, \quad \rho_{h_{1}}-\rho_{0}=\mathfrak{\beta}_{1}\left(\omega_{1}^{h_{1}-1} t^{\frac{1}{h_{1}}}\right) ; \\
\rho_{h_{1}+1}-\rho_{0}=\mathfrak{P}_{2}\left(t^{\frac{1}{h_{2}}}\right), \quad \rho_{h_{1}+2}-\rho_{0}=\mathfrak{P}_{2}\left(\omega_{2}^{1} t^{\frac{1}{h_{2}}}\right), \ldots, \rho_{h_{1}+h_{2}}-\rho_{0}=\mathfrak{P}_{2}\left(\omega_{2}^{h_{2}-1} t^{\frac{1}{h_{2}}}\right) ;
\end{array}
$$

geben. Dann besitzt die ursprüngliche Substitution, also die für welche $t=0$ gesetzt ist, für $\rho=\rho_{0}$ eine Kette der Ordnung $h_{1}$, eine zweite der Ordnung $h_{2}, u$. s. $f$. Die Entwickelungen der Wurzeln bestimmen also die Ordnungen der Ketten, bezw. der Elementarteiler.

\section{4.}

Zum Zwecke unserer weiteren Untersuchungen betrachten wir, wie auch schon im $\$ 2$, die Subdeterminanten $\Delta_{a, \beta}$ der Normalform unserer Substitution mit den Coefficienten $c_{x \lambda}$. Dabei bedeuten wie oben $\alpha, \beta$ die Indices der weggelassenen Zeile und Colonne. Um die Bezeichnung zu vereinfachen, nehmen wir $\rho=0$ als $m$-fache Wurzel von $\Delta(\rho)=0$ an und setzen die zugehörigen Zeilen in der Determinante an die erste Stelle.

Aus $\S 2$ können wir unmittelbar das Resultat übernehmen, dass $\Delta_{u, \beta}=0$ sei, wenn $a>\beta$ wird.

I. Nun setzen wir zunächst $\beta \leqq m$ voraus.

Ist $\alpha=\mathrm{I}, 2, \ldots, \beta$, so zerlegt sich $\Delta_{a \beta}$ sofort in das Product einer Determinante $(n-\mathbf{I})^{\text {ter }}$ Ordnung, die den $m$ ersten Zeilen, und einer solchen $(n-m)^{\text {ter }}$ Ordnung, die den $(n-m)$ letzten Zeilen von $\Delta$ entnommen ist. Die letzte sei

$$
A_{0}+A_{1} \rho+A_{2} \rho^{2}+\ldots+A_{n-m} \rho^{n-m}=T(\rho), \quad\left(A_{0} \neq 0\right) ;
$$

dass $A_{0} \neq 0$ sein muss, folgt leicht daraus, dass diese Hauptsubdeterminante die Wurzel $\rho=0$ nicht mehr besitzt. Der erste Factor ist aus den $m$ ersten Colonnen der $m$ ersten Zeilen derart entnommen, dass in ihnen $Z_{\alpha}, C_{\beta}$ unterdrückt wurden. Dabei sieht man sofort, wie aus den Zeilen $Z_{a+1}, Z_{a+2}, \ldots, Z_{\beta \rightarrow 1}$ die Glieder der Parallelreihe zur Haupt- 
Zur: Theorie der linearen Substitutionen.

diagonale, welche für die Substitution charakteristisch ist, in die Diagonale selbst rücken (vgl. $\S 2$ ), und so folgt für diesen Factor der Wert

$$
\pm \rho^{m-1-\alpha+\beta} \text { oder } o \text {, }
$$

je nachdem $Z_{\alpha}, C_{\beta}$ in dieselbe Kette, die zu $\rho=0$ gehört, einschneiden oder nicht.

Ist $\alpha>\beta$, so verschwindet $\Delta_{\alpha \beta}$ nach $\S 2$.

II. Ist $\beta>m$, so zerfällt die Determinante $\Delta_{a, 3}$ wieder in das Product zweier anderen, deren $z$ weite von der Ordnung $(n-m)$ ist und aus den letzten $(n-m)$ Zeilen entnommen wird. Da aber hier $C_{\beta}$ gestrichen ist, so wird aus den ersten Colonnen, die nur Nullen innerhalb der betreffenden letzten Zeilen enthalten, eine solche herausgenommen werden müssen. Es verschwindet daher $\Delta_{a, \beta}$.

Dies ergiebt

$$
\Delta_{a, \beta}= \pm A_{0} \rho^{m-1} \pm A_{1} \rho^{m} \pm \ldots, \quad(\alpha=\beta \leq m),
$$

$$
\Delta_{a, 3}= \pm \rho^{m-1-\alpha+\beta} . T(\rho) \text { oder }=0, \quad(\alpha<\beta \leq m)
$$

$$
\Delta_{a, 3}=0, \quad \text { (in allen anderen Fällen). }
$$

Geht man von dem eben betrachteten $\Delta$ zu dem im vorigen Paragrafen untersuchten benachbarten $\theta$ mit den $u m r_{x \lambda} . t$ vermehrten Elementen über, so folgt modulo $t$

$$
\theta_{\alpha \beta} \equiv \pm A_{0} \rho^{m-1} \pm A_{1} \rho^{m} \pm \ldots, \quad(\alpha=\beta \leq m),
$$

$$
\theta_{\alpha, \beta} \equiv \pm \rho^{m-1-\alpha+\beta} \cdot T(\rho) \text { oder } \equiv \mathrm{o}, \quad(\alpha<\beta \leqq m)
$$

$$
\theta_{\alpha \beta} \equiv \mathrm{o}, \quad \text { (in allen anderen Fallen). }
$$

Nun setzen wir

$$
\theta_{1 \alpha} \xi_{1}+\theta_{2 a} \xi_{2}+\ldots+\theta_{n a} \xi_{n}=\varphi_{a}(\xi)
$$

dann folgt aus der Fundamental-Eigenschaft der Determinanten und der Definition der Substitution mit der Determinante $\theta$ 


$$
\begin{aligned}
\varphi_{a}(X) & =\rho \varphi_{a}(x)+x_{a} \theta, \\
\varphi_{a}^{\prime}(X) & =\rho \varphi_{a}^{\prime}(x)+\varphi_{a}(x)+x_{a} \theta^{\prime}, \\
\varphi_{a}^{\prime \prime}(X) & =\rho \varphi_{a}^{\prime \prime}(x)+2 \varphi_{a}^{\prime}(x)+x_{u} \theta^{\prime \prime}, \\
\cdot & \cdot \cdot \cdot \cdot \cdot \cdot \cdot \cdot \cdot \cdot \cdot \cdot \cdot \\
\varphi_{a}^{(m-1)}(X) & =\rho \varphi_{a}^{(m-1)}(x)+(m-1) \varphi_{a}^{(m-2)}(x)+x_{a} \theta^{(m-1)},
\end{aligned}
$$

wobei die oberen Indices die Ableitungen der betreffenden Functionen nach $\rho$ bezeichnen sollen. $\varphi_{a}^{(m \cdots 1)}$ enthält als Coefficienten nach (4)

$$
\theta_{1 \alpha}^{(m-1)}, \theta_{2 \alpha}^{(m-1)}, \ldots, \theta_{a \alpha}^{(m-1)}, \ldots, \theta_{n o}^{(m-1)}
$$

Setzt man hierin für $t$ und $\rho$ jedesmal o ein, so verschwindet, wenn $\alpha>m$ ist, nach (3) jedesmal die ganze Reihe. Ist dagegen $\alpha<m$, so zeigt (3) dass $\theta_{a \alpha}^{(m-1)}$ sicher von Null verschicden ist, und möglicherweise auch noch die vorhergehenden Glieder der Zeile. (6), während die folgenden verschwinden.

Für $a<m$ ist also $\varphi_{a}^{(m-1)}(x)$ nicht gleich Null. Da $\rho=0$ eine $m$-fache Wurzel von $\Delta=0$ ist, so verschwinden $\theta^{(m-1)}, \theta^{(m-2)}, \ldots$ Die letzte Gleichung von (5) ergiebt also für $\rho=0, t=0$

$$
\varphi_{a}^{(m-1)}(X)=(m-1) \varphi_{a}^{(m-2)}(x) . \quad(t=0, \rho=0)
$$

Ist $\varphi_{a}^{(m-2)}(x)$ identisch gleich Null, so haben wir in $\varphi_{a}^{(m-1)}(x)$ eine $\mathrm{zu} \rho=0$ gehörige Normalcoordinate gefunden, wie die letzte Gleichung von (5) zeigt. Ist $\varphi_{a}^{(m-2)}(x)$ dagegen nicht identisch Null, so liefert die in (5) vorhergehende Gleichung

$$
\varphi_{a}^{(m-2)}(X)=(m-2) \varphi_{a}^{(m-3)}(x) . \quad \quad(t=0, p=0)
$$

Ist hier $\varphi_{a}^{(m-8)}(x)$ identisch Null, so hat man eine aus $\varphi_{a}^{(m-3)}(x)$ und $\varphi_{a}^{(m-2)}(x)$ bestehende Kette zweiter Ordnung crlangt, da der Factor $(m-2)$ nur formal aber nicht im Wesen eine Änderung hervorruft. Ist dagegen $\varphi_{a}^{(m-3)}(x)$ nicht identisch Null, so kommen wir in gleicher Weise zu einem dritten Kettengliede $u$. s. f. 
Zur Theorie der linearen Substitutionen.

Da endlich für $\alpha=1,2, \ldots, m$ alle $\varphi$ von einander verschieden sind, weil ja $\varphi_{a}(x)$ mit $\theta_{a a}^{(m-1)}$ abbricht, so ergiebt uns das Schema (5) für $t=0, \rho=0$ alle zu der Wurzel $\rho$ gehörigen Ketten.

Bemerken wir weiter, dass die $\varphi_{u}(x)$ als Normalcoordinaten direct aus der vorgelegten Substitution mit den Coefficienten $c_{\lambda, \mu}+\gamma_{\lambda \mu} t$ mit Hülfe der Subdeterminanten $(n-\mathrm{I})^{\text {ter }}$ Ordnung gebildet werden können, so erkennt man Folgendes:

Es sei eine Substitution mit den Coefficienten $c_{x \lambda}$ vorgelegt; wir bilden eine benachbarte Substitution mit den Coefficienten $c_{x \lambda}+\gamma_{x \lambda} t$, wobei $t$ eine betiebig kleine Variable und die $r_{x \lambda}$ Constanten bedeuten, die so gewählt sind, dass die zugehörige charakteristische Gleichung keine gleichen Wurzeln besitzt. Dagegen mag die charakteristische Gleichung der ersten Substitution die Wurzel $\rho=0$ genau $m$-fach besitzen, Dann werden $m$ Wurzeln der charakteristischen Gleichung der zweiten Substitution für $t=0$ auch gleich $\mathrm{o}$.

Wir bezeichnen nun mit

$$
\theta_{1 \alpha}, \theta_{2 \alpha}, \theta_{3 \alpha}, \ldots, \theta_{n \alpha}
$$

die Adjuncten der Elemente aus der $a^{\text {ten }}$ Colonne in

$$
\left|c_{x \lambda}+\gamma_{x \lambda} t-\varepsilon_{x \lambda} \cdot \rho\right|
$$

und mit $\varphi_{a}(x)$ die lineare Function

$$
\theta_{1 \alpha} x_{1}+\theta_{2 \alpha} x_{2}+\ldots+\theta_{n \alpha} x_{n}
$$

die Ableitungen derselben nach $\rho$ sollen der Reihe nach durch

$$
\frac{\partial \varphi_{a}(x)}{\partial \rho}=\varphi_{a}^{\prime}(x), \quad \frac{\partial^{2} \varphi_{a}(x)}{\partial \rho^{2}}=\varphi_{a}^{\prime \prime}(x), \ldots, \quad \frac{\partial^{m-1} \varphi_{a}(x)}{\partial \rho^{m-1}}=\varphi_{a}^{(m-1)}(x)
$$

bezeichnet werden.

Dann wirl für $t=0, \rho=0$ das letzte Glied $\varphi_{a}^{(m-1)}(x)$ der Zeile (6) verschwinden bei $n-m$ Werten von $\alpha$. Wïhlt man für einen der übrigen Werte von a ausser dem letzten, nicht verschwindendem Gliede noch diejenigen vorhergehenden, die gleichfalls nicht verschwinden, so bilden diese die Glieder einer Kette der Normalform in der durch die Glieder von (6) angegebenen 
E. Netto.

Reihenfolge; die nicht verschwindenden $\varphi_{a}^{(m-1)}(x)$ geben also die einzelnen letzten Kettenglieder.

Durch diese Darlegungen ist der Übergang von der Normalform einer nicht singulären Substitution zur Normalform einer benachbarten singulären geliefert.

Giessen d. 25. September I89 I. 